\section{An illusory motion of a stationary target during head motion is unaffected by paradoxical retinal motion: A reply to Shebilske and Proffitt (1983)}

\section{WALTER C. GOGEL University of California, Santa Barbara, California}

Shebilske and Proffitt (1983) criticize an experimental analysis by Gogel (1982) of the illusory motion concomitant with a motion of the head found for a binocularly viewed target presented in a dark surround. It is pointed out in the present reply that these criticisms are unfortunately the result of their misunderstanding the conditions used in the study. To make this clear, in the first part of this reply, the conditions of the study by Gogel (1982) are described, and equations, developed in that study and essential to the discussion, are listed. This is followed by a summary of the assumptions and arguments presented by Shebilske and Proffitt and a discussion as to why these are inappropriate to their conclusions. Finally, it is again noted that strong evidence is available to support the conclusion that apparent distance is important for the understanding of illusory object motion concomitant with a motion of the head.

\section{A Summary of "Analysis of the Perception of Motion Concomitant With a Lateral Motion of the Head"' (Gogel, 1982)}

The purpose of Experiment 1 in the study by Gogel (1982) was to test the assertion of Shebilske and Proffitt (1981) that the illusory motion of a physically stationary target, concomitant with a motion of the head, is paradoxical in the sense that, during this illusory motion, the target appears to move without going anywere. The results from Experiment 1 were opposed to this assertion in that apparent motion from real motion and from illusory motion were found to be algebraically additive. In that experiment, three values of physical concomitant motion (m) of the target (including a condition of zero motion) and two amounts of illusory concomitant motions $\left(\mathrm{m}^{\prime}\right)$ were used. The illusory concomitant motions were produced in the manner indicated in Figure 1. A point of light (target), always physically at $25 \mathrm{~cm}$ (D), was viewed binocularly while the head was moved left and right repetitively between Positions 1 and 2 through a distance K. Either a 25- or

The author would like to thank Jack Loomis for his helpful comments on an earlier version of this reply. The author's mailing address is: Department of Psychology, University of California, Santa Barbara, California 93106.
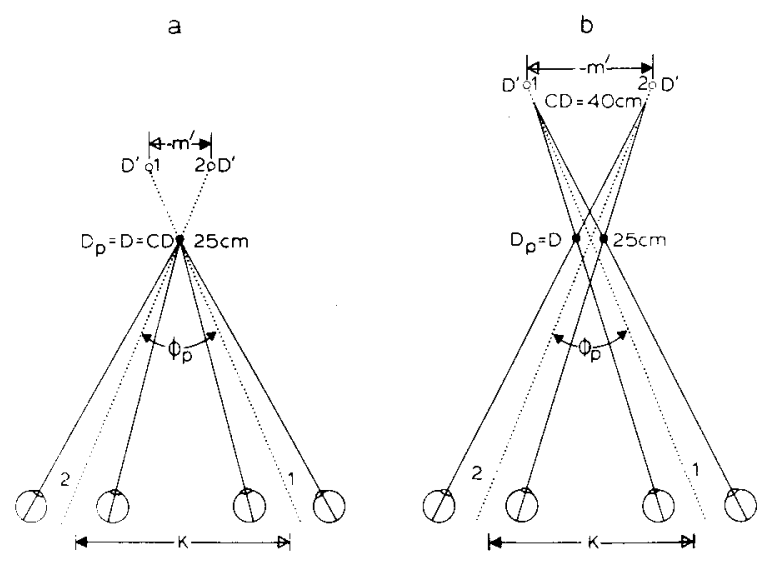

Figure 1. Schematic diagrams illustrating the presentation of the target (point of light) viewed binocularly, with the head moving left and right repetitively through a lateral distance, $\mathrm{K}$, in Experiment 1 of the study by Gogel (1982). In situations a and b, the point of light is physically a distance (D) of $25 \mathrm{~cm}$ from the observer. The distance to which the eyes must converge (CD) to binocularly fuse the point is $25 \mathrm{~cm}$ in situation a and $40 \mathrm{~cm}$ in situation $b$. The convergence to the target was modified by placing polarizing material on the display surface and in goggles worn by the observer, so that for the CD of $\mathbf{4 0} \mathrm{cm}$ the right point was seen only by the right eye and the left point only by the left eye (see Gogel, 1982, p. 243, for additional details). The dotted lines represent the direction from the cyclopean eye to the target at the two extremes of head motion. The position in space around which this direction pivots as the head is moved is called the pivot dis. tance $\left(D_{p}\right)$. In both situations, $D_{p}=D=25 \mathrm{~cm}$. The apparent distance ( $\left.D^{\prime}\right)$ of the target is greater than $D_{p}$, resulting in an apparent motion ( $\left.m^{\prime}\right)$ of the target concomitant with, but in a direction opposite to, the motion of the head. The $m$ ' is smaller in situation a than in situation b, according to the apparent-distance/ pivot-distance hypothesis, because the ratio $\mathbf{D}^{\prime} / \mathbf{D}_{\mathrm{p}}$ is greater in the latter case. The fovea on each eye is labeled $f$, with $t$ being the position of the image of the target on the eye. As indicated in the figure, in neither situation will the head motion result in a motion of the image of the target $(t)$ on the retina.

a 40-cm convergence distance (CD) of the eyes was necessary in order for the observer to fixate the point binocularly, with the target always presented in an otherwise dark surround. Figure la illustrates the situation in which CD was $25 \mathrm{~cm}$, and Figure $1 \mathrm{~b}$ the situation in which $C D$ was $40 \mathrm{~cm}$. To produce the convergence distance of $40 \mathrm{~cm}$ (Figure $1 \mathrm{~b}$ ), two laterally separated points of light, both physically at $25 \mathrm{~cm}$, were presented simultaneously. Using polarizing filters, the right point was imaged on the right eye only and the left point on the left eye only. Converging the eyes to the distance of the point resulted in a perception of one point of light only and, as shown, no motion of the point (target) was present on the retina as the head was moved in either Figure la or Figure $1 b$. The dotted lines in Figure 1 represent the directions from the cyclopean eye to the binocularly fused target for the extremes of the head motion. 
The horizontal lines labeled $\mathrm{m}^{\prime}$ are the apparent (illusory) motions of the target concomitant with the motion of the head. The hypothetical position around which the direction to the target from the cyclopean eye rotated as the head was moved is called the pivot distance, $D_{p}$, with $\phi_{p}$ the pivot angle. Because the greater $C D$ resulted in a greater perceived distance (D') of the target without changing $D_{p}$, the amount of illusory concomitant motion $\left(m^{\prime}\right)$ was greater in the situation of Figure $1 \mathrm{~b}$ than in that of Figure 1a, thus providing the two values of $\mathrm{m}^{\prime}$ used in the experiment. In both situations, as indicated by the arrows and by the negative sign of the $\mathrm{m}^{\prime}$, the illusory motion was opposite in direction to the direction of the head motion. It can be shown that the illusory motion of the target is specified geometrically by

$$
m^{\prime}=\mathbf{K}\left(1-D^{\prime} / D_{p}\right)
$$

with Equation 1 called the apparent-distance/pivotdistance hypothesis. According to this equation, for a given value of head motion, the perceived concomitant motion of the target will depend upon the ratio of the apparent distance to the pivot distance. Equation 1 assumes that the pivot angle and the head motion are correctly perceived, that is, that $\phi_{\mathrm{p}}^{\prime}=\phi_{\mathrm{p}}$ and $\mathbf{K}^{\prime}=\mathbf{K}$. These assumptions were validated in Experiment 1 . Although Figure 1 illustrates only situations in which the target is stationary $(m=0)$, the hypothesis also applies when $D_{p}$ is adjusted by physically moving the binocularly fused target laterally concomitant with the head motion. In the situation of Figure 1, moving the fused binocular target laterally in the direction of the head motion increases $D_{p}$, whereas the opposite motion of the target relative to the head motion decreases $D_{p}$. The effect of $m$ on $\mathrm{m}^{\prime}$ is given by

$$
\mathrm{m}^{\prime}=\left(\mathrm{KD}-\mathrm{KD}^{\prime}+\mathrm{mD}^{\prime}\right) / \mathrm{D}
$$

Thus, given the physical terms $K, D$, and $m$, and the perceived motion, $\mathrm{m}^{\prime}$, the perceived distance, $\mathrm{D}^{\prime}$, can be calculated from Equation 2 as follows:

$$
D^{\prime}=\mathrm{D}\left(K-\mathbf{m}^{\prime}\right) /(\mathrm{K}-\mathbf{m}) .
$$

If the pivot distance is adjusted until $D_{p}=D^{\prime}$, it follows from Equation 1 that $\mathrm{m}^{\prime}=0$, and hence from Equation 3 that

$$
D^{\prime}=K D /(K-m) .
$$

According to Equation 4, the physical motion, $m$, of the target required to make $m^{\prime}=0$ provides a measure of the $\mathbf{D}^{\prime}$ of the target. This method of measuring perceived distance is called the null procedure. Thus, by applying the apparent-distance/pivotdistance hypothesis, $D^{\prime}$ can be measured by measuring $\mathrm{m}^{\prime}$ and applying Equation 3, or by nulling out the apparent concomitant motion ( $\left.m^{\prime}\right)$ and applying Equation 4. Conversely, the validity of Equations 2,3 , and 4 provides support for the apparent-distance/ pivot-distance hypothesis.

Three methods of measuring the perceived distance of the target were used in Experiment 1. In one, a direct measure of $\mathrm{D}^{\prime}$, the observer pointed with an unseen hand to the apparent distance of the target. Another method measured $D^{\prime}$ indirectly by directly measuring $\mathrm{m}^{\prime}$ and applying Equation 3, with $\mathrm{m}^{\prime} \mathrm{ob}-$ tained either by pointing to the right and left extremes of the apparent motion or by adjusting the separation of two rods by touch, to duplicate the lateral extent of the perceived motion (the hand adjustment procedure). A second indirect method was to null the apparent concomitant motion by a physical concomitant motion (m) in the opposite direction. From the $m$ required in order for the apparent concomitant motion of the target to disappear $\left(\mathrm{m}^{\prime}=0\right), \mathrm{D}^{\prime}$ was computed using Equation 4 . The $D^{\prime}$ obtained by the null procedure was usually larger than that obtained from the other procedures.

In Experiment 2, the point stimulus was always physically stationary at $25 \mathrm{~cm}$ and the convergence was to either 25 or $40 \mathrm{~cm}$, as shown in Figure 1. The only measure of $\mathrm{m}^{\prime}$, and thus indirectly of $\mathrm{D}^{\prime}$, that was obtained used the hand adjustment procedure. The main purpose of Experiment 2 was to determine whether the error in perceived distance shown in Figure la could be explained by an error in convergence in fixating the 25-cm distance (a fixation disparity). According to Gogel and Tietz (1973), the illusory concomitant motion found in this case can be attributed to the specific distance tendency, which is the tendency in the absence of completely effective cues of distance for perceived distance to be modified toward a distance of 2 or $3 \mathrm{~m}$ from the observer. An alternative interpretation proposed by Owens and Leibowitz (1976) is that the error is the result of an error in fixation (a fixation disparity) in the direction of the resting state of convergence (a distance of about $1.2 \mathrm{~m}$ ). Fixation disparity was measured in Experiment 2 using a nonius technique. It was found that errors in converging to either the $25-\mathrm{cm}$ (Figure $1 \mathrm{a}$ ) or the $40-\mathrm{cm}$ (Figure $1 \mathrm{~b}$ ) distance were small (average values of 5.2 and $2.7 \mathrm{~min}$ of arc, respectively) and were far too small to explain the values of $\mathrm{m}^{\prime}$ obtained in the situation of Figure 1a of Experiment 1.

\section{Summary of the Comments by Shebilske and Proffitt (1983) Entitled 'Paradoxical Retinal Motions During Head Movements: Apparent Motion Without Equivalent Apparent Displacement"}

Shebilske and Proffitt argue for an interpretation of some of the results obtained in the above study as an instance of the phenomenon of paradoxical motion. They suggest that there are two kinds of motion effects that can occur concomitant with head 
motion. One of these is nonparadoxical motion that is accompanied by apparent displacement of the target. This motion, they suggest, is measured by the procedure in which the observer points beneath the apparent position of the target at each extreme of head motion and also by the hand adjustment procedure in which the observer adjusts the separation of two posts by touch to equal the extent of lateral motion perceived in the target. The other kind of apparent motion effect that was present in the study by Gogel (1982), according to Shebilske and Proffitt, is a consequence of the presence of a retinal image motion (RIM) of the target concomitant with the motion of the head. This concomitant retinal motion is considered by these authors to produce a paradoxical motion of the target characterized as an apparent motion without a change in apparent position. They note two aspects of this paradoxical motion which they regard as important. One is that, because the paradoxical motion does not produce apparent displacement of the target, it is expected to have no effect upon the displacement measures of $m^{\prime}$ involving pointing or the hand adjustment procedure. But, according to these authors, paradoxical motion is an apparent motion and it will modify the results from the null procedure. The second characteristic is that RIM concomitant with head motion is assumed to have been present in the situation of Figure $1 \mathrm{~b}$ above but not in the situation of Figure 1a. Shebilske and Proffitt (1983) state: "Thus, retinal image movements will occur even with a single target if an observer converges his or her eyes in front or in back of the target"'(p. 476). On this basis, Shebilske and Proffitt expect that when CD $>D$ (Figure $1 b$ ) the null measure of $D^{\prime}$ (applying Equation 4) will be larger than displacement measures of $D^{\prime}$ (applying Equation 3). On the other hand, they expect that no difference in these measures will be found when $\mathrm{CD}=\mathrm{D}$ (Figure 1a). It follows that to achieve a correct measure of $D^{\prime}$ using the null procedure with $C D \neq D$, the contribution of RIM to the null adjustment defined by Equation 4 needs to be removed. According to Shebilske and Proffitt, this can be achieved by adding a correction term to Equation 4, as shown in their Equation 11. Using these concepts, they then proceed to compare the $D^{\prime}$ results obtained in Experiment 1 of Gogel (1982) from the situation in which the apparent displacement of the physically stationary target was measured with those obtained from the situation in which the target was moved concomitantly with the head so as to achieve the null criterion. They find that the difference between the null and displacement measures of $D^{\prime}$ do not reach significance (using the $\mathrm{p}<.01$ criterion) for $C D$ of $25 \mathrm{~cm}$, but are significant for $C D=40 \mathrm{~cm}$. They interpret these results as providing evidence for the paradoxical-motion hypothesis. In their Figures 2 and 3, Shebilske and Proffitt attempt to calculate the factor needed to correct Equation 4. Thus, Shebilske and Proffitt concluded that they had identified a factor (RIM) that could contribute to apparent illusory motion in addition to the factors of apparent distance and pivot distance specified by the apparent-distance/pivot-distance hypothesis. As indicated, this factor is expected to result in an error in the measurement of perceived distance only when the null procedure is used.

\section{An Evaluation of the Comments by Shebilske and Proffitt}

The objections to the apparent-distance/pivotdistance hypothesis expressed by Shebilske and Proffitt are limited to a proposed correction term for Equation 4, leaving Equations 1, 2, and 3 intact. Sometimes, however, they write as if this limited correction permitted them to reject the apparent-distance/pivotdistance hypothesis in its entirety. For instance, in discussing the results in their Table 1 (1983), compiled from Experiment 1 of Gogel (1982), they state: "The results clearly oppose the apparent-distance/ pivot-distance hypothesis and strongly support the paradoxical-motion hypothesis" (p. 479). This conclusion is both overstated and unsupported. Shebilske and Proffitt are explicit in expecting RIM to occur only for the situation in which the convergence distance $(40 \mathrm{~cm})$ is greater than the physical distance $(25 \mathrm{~cm})$. But, as indicated by Figure 1 above, there is no more reason to postulate the presence of RIM concomitant with head motion in the situation of Figure $1 b$ than in the situation of Figure $1 a$ and no reason to expect that a RIM concomitant with head motion occurred in either case. Shebilske and Proffitt discuss their assumptions regarding RIM in relation to their Figure 1. Their Figure 1A is intended to represent the same situation as Figure $1 \mathrm{~b}$ of the present article. As indicated by the previous quotation from Shebilske and Proffitt, they expect RIM (defined as $\phi_{2}-\phi_{1}$ in their Figure 1) to occur whenever CD $>D$, with only the null method of measuring $D^{\prime}$ expected to reflect the paradoxical motion. According to Figure $1 \mathrm{~b}$ in the present article, there was no RIM when $C D>D$ in Experiment 1 of the Gogel (1982) study either for a stationary or for a moving target, and therefore, no basis for their conclusion regarding the effect of the paradoxical motion assumed to result from RIM on the data of their Table 1.

It is of interest to examine the conditions that might be thought to produce some retinal image motion as the head is moved while a single binocular object is being viewed. Figure $2 a$ illustrates a factor that did not occur in the study by Gogel (1982), as indicated by the nonius measures, that is, a substantial fixation or convergence disparity. Again, the dotted lines represent the direction from the cyclopean eye to the target, with the fovea labeled $f$ and the image of the target labeled $t$. The fixation disparity illustrated 

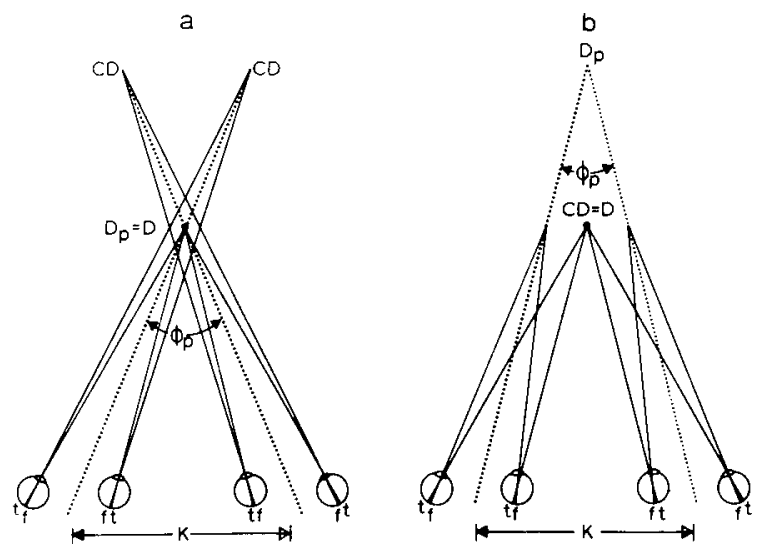

Figure 2. Schematic diagrams representing a convergence disparity (a fixation disparity) in Drawing a and a pivot disparity in Drawing b while binocularly viewing a point of light (target) as the head is moved left and right repetitively through a lateral distance $(K)$. The foven of each eye is labeled $f$, with t the position of the image of the target on the eye. The dotted lines represent the intersection of directions at the extremes of head motion from the cyclopean eye to a position in space. The distance in space at which these directions intersect is colled the pivot distance. The pivot distance is at the target in Drawing a and at a position in space beyond the target in Drawing $b$. Although a fixation disparity (Drawing a) results in an opposite displacement of $t$ in the two eyes, there is no motion of $t$ in this case in either eye as the head is moved. On the other hand, in Drawing $b$, a motion of $t$ that is the same on each retina does occur as a result of the head movement. From nonius measures, a substantial fixation disparity was not present in the Gogel (1982) study and a substantial pivot disparity was very unlikely to have occurred in the absence of a second object at the far distance to determine the increased $D_{p}$.

is to a distance behind the target. It will be noted that $t$ is displaced to the right of $f$ in the right eye and to the left of $f$ in the left eye for all positions of the head. There is no motion of $t$ across the retina as the head is moved laterally. A fixation disparity does not produce a RIM.

Figure 2b illustrates a situation in which there is a fixation error of another kind, which can be called a pivot disparity. In the case illustrated, the position in space around which the direction from the cyclopean eye to the target pivots as the head is moved is at a greater distance than $\mathrm{D}$ or $\mathrm{CD}$. As indicated by the changed relationship between $f$ and $t$ as the head is moved laterally, a pivot disparity produces a retinal image motion. However, the possibility is remote that a substantial pivot disparity occurs frequently or occurred in the study by Gogel (1982). In order for this to happen, the observer would need to systematically shift the direction of cyclopean gaze to the right and left of the target in phase with the head motion so as to define a pivot position in empty space. Since this would involve a displacement of cyclopean gaze from the direction of the target, it is expected, from a study by Frances and Owens (1983), that a substantial pivot disparity would be accompanied by a substantial fixation disparity in the direction of the resting stage of convergence. But, a substantial fixation disparity did not occur in the study by Gogel (1982); nor was the small fixation disparity that did occur in the situation of Figure $1 \mathrm{~b}$ in the direction expected from the resting state. In summary, there is no reason whatsoever to expect that RIM concomitant with a motion of the head was present in any of the situations of Experiment 1. In particular, the notion of Shebilske and Proffitt that RIM must occur whenever $C D$ differs from $D$ for a single target in an otherwise dark visual field is incorrect. As has been discussed in relation to Figure $2 b$ above, a pivot disparity, not a convergence disparity, is required in order for an RIM to be present under these conditions. Finally, as will be discussed, even in the unlikely event that a pivot disparity of an appreciable magnitude had been present, there would be no reason to suppose that the RIM would result in a component of apparent motion, paradoxical or otherwise.

The previously cited conclusion by Shebilske and Proffitt (1983), that the data of their Table 1 provides strong support for the paradoxical-motion hypothesis and clear opposition to the apparent-distance/ pivot-distance hypothesis, in addition to being unwarranted, is exaggerated in two ways. First of all, as discussed, their criticisms apply only to a modification of Equation 4 and are not a rejection of the apparent-distance/pivot-distance hypothesis. Although they argue (on mistaken grounds) that Equation 4 requires a correction for paradoxical motion in order to provide an unbiased measure of $D^{\prime}$, they do not advocate a correction when direct measures of $\mathrm{m}^{\prime}$ are used to measure $\mathrm{D}^{\prime}$ by applying Equation 3 . But, if Equation 3 is valid, together with the assumptions that $\phi_{p}^{\prime}=\phi_{p}$ and $K^{\prime}=K$, it follows that Equation 1 also must be valid. Whatever the reason for the larger values of $D^{\prime}$ obtained from the null procedure as compared with the procedure of directly measuring $\mathrm{m}^{\prime}$, it is not the result of concomitant RIM. The question of whether the measures of $D^{\prime}$ from Equations 3 and 4 do indeed differ should not mask the fact that both provide measures of $D^{\prime}$ that are a monotonic increasing function and possibly a linear function of the distance actually perceived. In studies in which these equations have been or are likely to be applied (see Gogel, 1981), more stringent assumptions are seldom required. Another reason to be skeptical regarding the above conclusion by Shebilske and Proffitt is statistical. Although the difference between the null and displacement measures of $D^{\prime}$ in their Table 1 did not reach the .01 level for the case in which $C D$ and $D$ were both at $25 \mathrm{~cm}$, in that situation, five of the six observers showed a difference in the same direction as that obtained with the $40-\mathrm{cm}$ CD. 
Although the possibility is remote that RIM was a significant factor in the Gogel (1982) study, RIM certainly occurs in studies in which two objects are present at different distances and are viewed with a moving head. This is similar to the case of pivot disparity illustrated in Figure $2 b$, but with a second object available to provide a pivot distance different from that of the target. An instance of this situation is the viewing of two stationary fingers at different physical distances while the head is moved laterally and the near finger is fixated. In this case, the image of the near finger is always on the fovea as the head is moved, whereas the image of the far finger will move across the retina. This, according to Shebilske and Proffitt, should produce paradoxical motion that must be cancelled by a physical motion of the far finger in an opposite direction before the null criterion of no apparent motion of the far finger can be achieved. In two studies of this type of situation, a null adjustment procedure was used to measure $\mathbf{D}^{\prime}$ with fixation either on the target or on a different object at a greater or lesser distance than the target (Gogel, 1979; Gogel \& Tietz, 1977). It can be shown geometrically that complete cancellation of the targetimage motion on the retina would have resulted in a calculated value of $D^{\prime}$ equal to the physical distance of the fixated object. Although errors in the perceived distance of the target, as expected from the research of Wist and Summons (1976), occurred in the direction of the distance of fixation, substantial retinal motions of the target remained when the null criterion was achieved, particularly with the near distance of fixation. In other words, the null criterion was reached without the cancellation of what should have been, according to Shebilske and Proffitt, obvious amounts of paradoxical target motion concomitant with head motion. It seems, in opposition to the paradoxical-motion hypothesis of Shebilske and Proffitt, that RIM concomitant with head motion does not necessarily result in an apparent motion of any type.

In addition to applying to the case of a single object presented in a frontoparallel plane, the apparentdistance/pivot-distance hypothesis can be applied to situations in which several objects distributed in depth or a single object extended in depth is viewed while moving the head (see Gogel, 1981). The apparent concomitant relative motion $\left(\mathrm{m}_{\mathrm{n}}^{\prime}-\mathrm{m}_{\mathrm{f}}^{\prime}\right)$ of two objects $\mathrm{n}$ and $\mathrm{f}$ (one at a near and the other at $\mathrm{a}$ farther distance) can be expressed using Equation 2. If the objects are physically stationary $\left(m_{n}=m_{f}=0\right)$, it follows that

$m_{n}^{\prime}-m_{f}^{\prime}=\left(K / D_{f}\right) D_{f}^{\prime}-\left(K / D_{n}\right) D_{n}^{\prime}=\phi_{f} D_{f}^{\prime}-\phi_{n} D_{n}^{\prime}$

(Gogel, 1980), where $\phi_{\mathrm{n}}$ and $\phi_{\mathrm{f}}$ are the pivot angles expressed in radians. If $D_{f}^{\prime}=D_{f}$ and $D_{n}^{\prime}=D_{n}$, that is, if there is no error in the perceived distance of either object, then $m_{n}^{\prime}-m_{f}^{\prime}=0$ and the objects will not appear to move relative to each other, even though obviously there is relative image motion on the retina. According to the apparent-distance/pivot-distance hypothesis, under conditions in which the depth between the objects is correctly perceived, a relative RIM will occur without producing paradoxical motion or, indeed, apparent motion of any sort. On the other hand, if an error in either $D_{n}^{\prime}$ or $D_{f}^{\prime}$ occurs, illusory concomitant motion will be present, as indicated by Equation 5 .

Although the paradoxical-motion hypothesis of Shebilske and Proffitt does not provide a viable alternative to the apparent-distance/pivot-distance hypothesis, there is one illusory concomitant motion described by Shebilske and Proffitt which is not likely to be explained entirely by errors in perceived distance. For an observer riding in a moving vehicle, nearby stationary objects often appear to move rapidly in a direction opposite to the motion of the vehicle. To explain this in terms of errors in perceived distance, it would be necessary to suppose that the perceived distance of the nearby objects was greatly overestimated. A more plausible explanation that also is consistent with the apparent-distance/pivotdistance hypothesis is available. It will be recalled that the application of this hypothesis in Equations 1-4 involves two assumptions. One is that the visual direction from the observer to the target is correctly perceived $\left(\phi_{p}^{\prime}=\phi_{p}\right)$. The other is that the observer can sense correctly the motion of his head $\left(K^{\prime}=K\right)$. According to the results of the 1982 study by Gogel, both assumptions are justified under conditions which include an active, but probably not too rapid, motion of the head. With the passive motion of the head that occurs when riding in a moving vehicle, the proprioceptive or other nonvisual information normally available for sensing the motion of the head is reduced or largely absent and, in an extreme case, all sensory support for the perception of the motion of the head is lost. But, since the observer continues to be able to correctly perceive the direction of objects from the head, nearby objects appear to move opposite to the observer's motion. A similar illusory perception of motion occurs if an observer is riding in a slowly moving train which appears to him to be stationary. In this case, a nearby train that physically is stationary will appear to move in the opposite direction. From the 1982 study by Gogel, however, for the situation in which two fingers are held at different distances and are viewed while voluntarily moving the head laterally, the assumption that $K^{\prime}=K$ is likely to be valid. The illusory relative motion in this case can be attributed to an error in the perceived depth between the fingers.

In referring to the possibility of developing an inclusive theory of motion perception, Shebilske and Proffitt (1983) state: "If such a theory is ever put 
forth, it will probably not include the general assertion of the priority of perceived distance for perceiving motion, since the present analysis suggests that perceived distance is not always involved in the apparent concomitant motion of physically stationary objects" (p. 481). In view of the flaws in their analysis, this conclusion is left unsupported. It is based, unfortunately, on a misunderstanding both of when a retinal motion of the target image is likely to occur and of the consequences of this retinal motion for perceived motion when it does occur. It follows that their analysis does not challenge the importance of apparent distance in the perception of illusory motion concomitant with a motion of the head. The importance of this latter factor has been demonstrated in a series of studies using a single target, several targets at different distances, or a target extended in depth (see Gogel, 1981, for a review of these studies). For example, when retinal motion is absent or held constant, variations in perceived distance or depth have been shown to produce a marked effect upon the magnitude and the direction of the motion perceived. The reader can readily experience an illusory concomitant motion, produced by an error in perceived depth and occurring in the absence of retinal motion, by moving the head laterally while viewing a stereogram physically drawn on a sheet of paper. This accumulated evidence, although largely ignored by Shebilske and Proffitt in their comments, provides strong support for the role of apparent distance in illusory motion concomitant with a motion of the head.

\section{REFERENCES}

Frances, E. L., \& Owens, D. A. The accuracy of binocular vergence for peripheral stimuli. Vision Research, 1983, 23, 13-19.

GoGEL, W. C. The common occurrence of errors of perceived distance. Perception \& Psychophysics, 1979, 25, 2-11.

GogeL, W. C. The sensing of retinal motion. Perception \& Psychophysics, 1980, 28, 155-163.

GOGEL, W. C. Perceived depth is a necessary factor in apparent motion concomitant with head motion: A reply to Shebilske and Proffitt. Perception \& Psychophysics, 1981, 29, 173-177.

GOGEL, W. C. Analysis of the perception of motion concomitant with a lateral motion of the head. Perception \& Psychophysics, 1982, 32, 241-250.

GogeL, W. C., \& Tietz, J. D. Absolute motion parallax and the specific distance tendency. Perception \& Psychophysics, 1973, 13, 284-292.

Goaet, W. C., \& TIETz, J. D. Eye fixation and attention as modifiers of perceived distance. Perceptual and Motor Skills, 1977, 45, 343-362.

Owens, D. A., \& Leibowitz, H. W. Oculomotor adjustments in darkness and the Specific Distance Tendency. Perception \& Psychophysics, 1976, 20, 2-9.

Shenilske, W. L., \& Proffit, D. R. The priority of perceived distance for perceiving motion has not been demonstrated: Critical comments on Gogel's "The sensing of retinal motion." Perception \& Psychophysics, 1981, 29, 170-172.

Shemilske, W. L., \& Proffitr, D. R. Paradoxical retinal motions during head movements: Apparent motion without equivalent apparent displacement. Perception \& Psychophysics, 1983 , 34, 476-481.

Wist, E. R., \& Summons, E. Spatial and fixation conditions affecting the temporal course of change in perceived relative distance. Psychological Research, 1976, 39, 99-112.

(Manuscript received August 1, 1983; accepted for publication August 5, 1983.) 